N.M. LITCHINITSER ${ }^{1,2,}$

E. POLIAKOV ${ }^{3}$

\title{
Antiresonant guiding microstructured optical fibers for sensing applications
}

\author{
${ }^{1}$ OFS Laboratories, 19 Schoolhouse Rd., Somerset, New Jersey 08873, USA \\ ${ }^{2}$ Department of Electrical Engineering and Computer Science, University of Michigan, 1301 Beal Ave., \\ Ann Arbor, Michigan 48109, USA \\ ${ }^{3}$ National Research Council, Washington DC/AMSAM-RD-WS-ST, Bldg. 7804, 4 Redstone Arsenal, \\ Huntsville, AL 35802
}

\section{Received: 14 February 2005 / Revised version: 3 May 2005 Published online: 15 July 2005 • C Springer-Verlag 2005}

ABSTRACT A novel refractometric sensor utilizing unique spectral properties of antiresonant-guiding microstructured optical fibers is proposed. The sensor operation is based on the wavelength shift of the transmission spectrum in response to the refractive index change of a sample loaded in the airholes of the microstructured optical fiber. Refractive index changes on the order of $0.1 \%$ can be detected using less than a nanoliter of a sample.

PACS 42.81Qb; 42.70Qs; 42.81Pa

1

\section{Introduction}

Over the last two decades optical fibers and waveguides have been widely used for developing optical sensors [1]. Applications of fiber-based sensors range from environmental monitoring, aerospace and security to biology and medicine. Fiber optic sensors rely on a variety of physical properties of a sample under investigation or of the waveguide itself, including absorption, fluorescence, evanescent waves, plasmonic resonances, Bragg reflection and cladding mode coupling in long-period gratings [1-3]. Depending on a particular physical mechanism, different device requirements may apply such as sufficient interaction length and large overlap between light and a sample (analyte) in the case of absorptionbased sensors and the presence of the reference arm in the case of phase-shift-based sensors. The performance of the device is typically characterized by its sensitivity, operation range, necessary volume of the analyte and the overall complexity of the design.

Emergence of microstructured optical fibers (MOFs) opened up new opportunities for developing novel sensor designs. Microstructrured optical fibers are typically dielectric cylindrical waveguides with a cross-section containing a structure on the scale of a micron and they are invariant in longitudinal direction [4-8]. Several types of MOF-based sensors have been discussed in the literature. The first cat-

Fax: +1-734-647-2718, E-mail: natashan@eecs.umich.edu egory of MOF-based sensors is represented by evanescent wave devices. In these devices light is guided through a modified total internal reflection (TIR) in a solid (high refractive index) core. Evanescent field effects are exploited for sensing gases or liquids inside the air holes in the cladding or on the silica-air surface [9-14]. The sensitivity of this technique depends on the overlap between the light beam and the analyte. Overlap is defined as a fraction of power that is exposed to the analyte [14]. It has been shown that the overlap can be improved to some degree by increasing the ratio of the air-hole diameter to the lattice constant and by operating at longer wavelengths. However, there is a trade-off between the ratio of the optical wavelength to the lattice constant and the confinement loss, imposing limitations on maximum overlap that can be achieved. Also, since light confinement in the solid core of the waveguide relies on the mechanism of total internal reflection, the refractive index of the waveguide material sets the upper limit on the refractive indexes that can be measured. The second category of MOF-based sensors that was proposed recently utilizes hollow-core fibers [14, 15]. Both photonic bandgap guiding and TIR guiding hollow-core fibers have been explored for sensing applications. Recently, three-material hollow-core MOFs were proposed for sensing in aqueous solutions [14]. In this type of sensors the aqueous solution occupies the central core air hole, thus maximizing the overlap between the light beam and the analyte offering significant advantages over the evanescent type of sensors. It was shown that three-material MOF can be designed to guide through TIR by carefully choosing the air-fill fraction in the cladding such that the 'average' index of the air-silica cladding is below the refractive index of the particular analyte. In spite of the design differences, both evanescent-wave-based and three-material-based MOF sensors often rely on long interaction lengths of the light and the analyte. Thus confinement loss should be minimized in order to achieve good performance.

In this paper, we propose a compact all-fiber sensor utilizing unique spectral properties of MOF with localized high-index inclusions. The sensor operation is based on the wavelength shift of the transmission spectrum in response to the refractive index change of the analyte. We use a simple analytical model and numerical analysis to characterize the sensor performance. 
2

\section{Transmission properties of MOFs with localized} high-index inclusions

Recently, MOFs consisting of a low-index solid core surrounded by localized high-index inclusions have attracted significant attention due to their peculiar transmission properties [16-29]. Various configurations of MOF with localized high-index inclusions have been demonstrated. These include MOFs infiltrated with high-index Cargille liquid [16, 19, 26, 30], liquid-crystal-filled MOFs [20, 21], all-solid MOFs made of two different glasses [23-25]. Several potential applications including tunable filters [18], optical modulators and biosensors based on liquid-crystal-filled MOF [20] have been proposed.

The cross-section of a typical MOF consisting of a lowindex core surrounded by two ring of circular air-holes of diameter $d_{\mathrm{MOF}}$ filled with high refractive index $n$ material is shown in Fig. 1a. Other parameters of the MOF include core diameter $D$, spacing between the air-holes (lattice constant) $\Lambda$, refractive index of the core and the background cladding material (silica) $n_{0}$. The refractive index profile of this fiber along $x$ direction is shown in Fig. 1b. We have previously shown that many of the spectral properties of full threedimensional MOF structure can be understood by modeling light propagation in an equivalent planar two-dimensional waveguide [27-29]. Figure 2 shows calculated spectra for a two-dimensional planar waveguide with a refractive index profile shown in Fig. 1 for two values of the refractive index of high-index layers $n=1.78$ and $n=1.8$. In these simulations we used the following parameters: refractive index of silica core $n_{0}=1.44$, thickness of a high-index layer of the planar structure $d_{\mathrm{pl}}=3.62 \mu \mathrm{m}$, core size $D=12.2 \mu \mathrm{m}$, lattice constant $\Lambda=8 \mu \mathrm{m}$, and waveguide length $L=1 \mathrm{~cm}$.

(a)

(b)
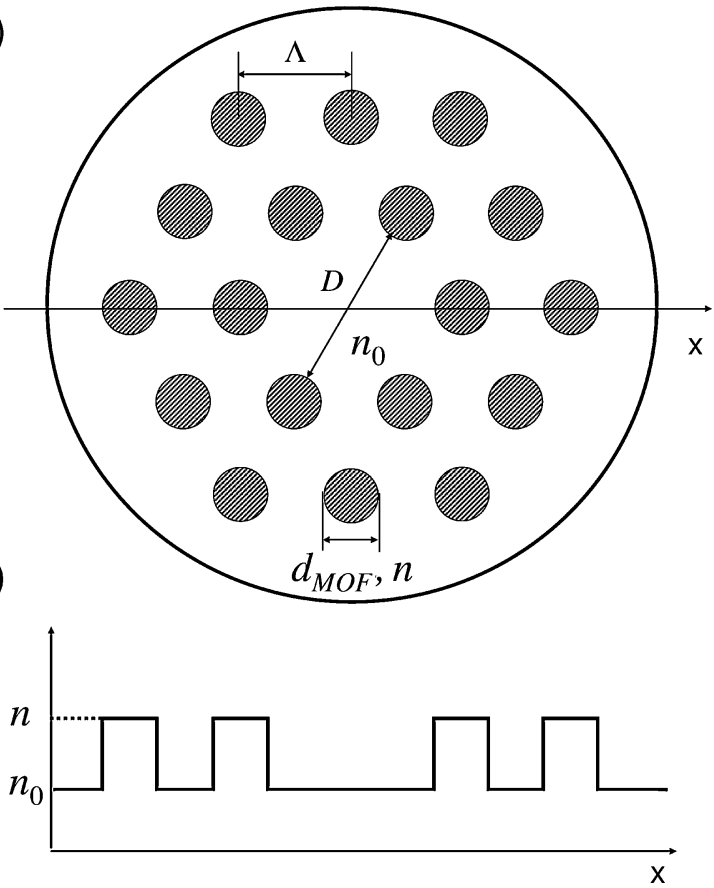

FIGURE 1 Transverse cross-section of the microstructured optical fiber with low-index core and two rings of high-index inclusions a, Corresponding refractive index profile along $x$ axis $\mathbf{b}$

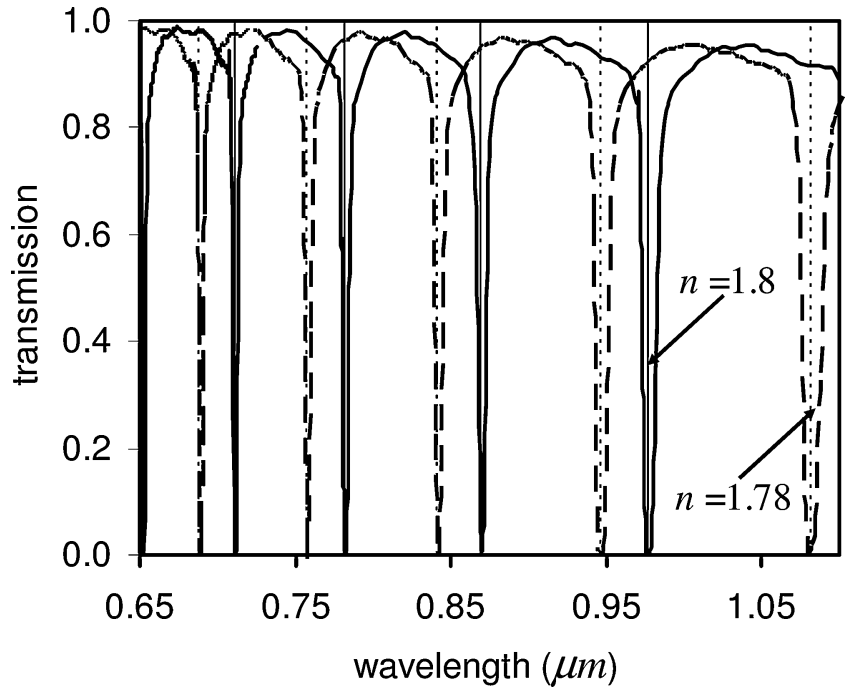

FIGURE 2 Calculated transmission spectra for two-dimensional waveguide with the refractive index profile shown in Fig. 1b for two values of the refractive index of the material infiltrated in the air holes: $n=1.8$ and $n=1.78$. Straight lines correspond to the analytical predictions using Eq. (1) solid line corresponds to $n=1.8$, dashed line corresponds to $n=1.78$ )

Propagation of light in the low-index core of MOFs with light-index inclusions has been explained in terms of an antiresonant reflecting optical waveguide (ARROW) $[17,27,31]$. Antiresonant guidance can be understood using a planar analog of MOF in which high-index cylindrical inclusions are replaced by high-index layers. Each high-index layer can be considered as a Fabry-Perot-like resonator. At a particular resonance the high-index layer becomes transparent and light escapes from the core resulting in a transmission spectrum minimum. Light is confined in the low-index core by antiresonant reflection from high-index layers.

The resonant condition for a high-index layer is given by $k_{\perp} d_{\mathrm{pl}}=m \pi$, where $k_{\perp}=2 \pi \lambda^{-1} \sqrt{n^{2}-n_{0}^{2}}$ is a transverse $k$-vector, $d_{\mathrm{pl}}$ is the width of the high-index layer of the planar structure, $m=1,2, \ldots$ is the resonance number, $n_{0}$ is the background (low) refractive index, $n$ is the refractive index of the material in the air holes (high-index material). Assuming that the effective refractive index of the core mode $n_{\text {eff }} \cong n_{0}$ (because $\lambda / D \ll 1$, where $\lambda$ is the wavelength of light), the wavelengths corresponding to transmission minima are given by:

$\lambda_{\mathrm{r}}=\frac{2 d_{\mathrm{pl}}}{m} \sqrt{n^{2}-n_{0}^{2}}$.

Straight lines in Fig. 2 correspond to resonant wavelengths predicted by Eq. (1).

Analog of ARROW regime of guidance also exists in MOFs. Resonant wavelengths corresponding to transmission minima in MOFs' spectrum can be written as:

$\lambda_{\mathrm{r}}=\frac{2 d_{\mathrm{MOF}}}{m+1 / 2} \sqrt{n^{2}-n_{0}^{2}}$

where $d_{\mathrm{MOF}}$ is a diameter of a high-index cylindrical inclusion. Equation (2) can be derived by considering each individual high-index cylinder as a step-index waveguide with a number of modes associated with it [17]. Resonant conditions (2) correspond to the modal cutoffs of the step-index waveguide. 
These cutoffs can be related to the scattering resonances associated with a plane wave impinging a high-index cylinder at a glancing angle [29]. The accuracy of the analytical model (1)-(2) was verified by numerical simulations [27-29] and in laboratory experiments [19, 21, 24, 25]. Good agreement between the analytical predictions, numerical simulations and experimental results in terms of the positions of high- and low-transmission regions even in short pieces $(500 \mu \mathrm{m}$ or less) of ARROW-MOF was demonstrated in [19]. Experimental studies of light transmission through longer lengths (up to 10 meters) of MOF with high-index inclusions confirming the ARROW mechanism of guidance have been reported in $[24,25]$.

Although the simple analytical model for ARROW regime in MOFs does not provide any predictions regarding the loss properties of the MOF, some estimations can be made based on analytical results for cylindrical hollow-core waveguide [32], planar and cylindrical (concentric) ARROW structure $[33,34]$, and qualitative description of the loss mechanism in MOFs [22]. Otherwise, loss can be calculated numerically $[19,29]$. It is noteworthy that ARROW model predicts the existence of the discrete transmission regions no matter how small refractive index difference $\Delta n=n-n_{0}$ is. Indeed, light propagation in MOF with localized inclusions and refractive index contrast of one percent has been recently demonstrated [23, 24]. White et al. showed using the multipole method that ARROW mode loss in transmission regions can be reduced by several orders of magnitude by adding more layers of high-index inclusions [29].

Main characteristics of light propagation in MOFs with high refractive index localized inclusions can be summarized as follows: (i) transmission spectra of these fibers consist of multiple bands of high and low transmission [17-25, 27-29]; (ii) even one ring of high-index inclusions is enough to confine light in the low index core, although losses decrease as more rings added [24, 27-29]; (iii) dispersion changes rapidly at wavelengths close to the transmission minima, a feature typical for many resonant structures, and changes sign within each high transmission region [26, 29]. One of the most interesting properties of MOF with high refractive index inclusions is that the locations of transmission minima are largely determined by the inclusions' diameter and the refractive index, and not by their separation, core size and number of inclusions. Indeed, Fig. 2 shows that transmission minima shift significantly ( $\sim 20-40 \mathrm{~nm}$ depending on resonance number $m$ ) as $n$ changes by only $1 \%$. Depending on the properties of a particular highindex material, refractive index $n$ changes in response to a variety of physical parameters including temperature, electric or magnetic field, humidity, etc. Corresponding wavelength shift of the transmission resonances occurring as a result of the refractive index change of the material inside the air-holes provides an opportunity for designing a new type of sensor.

3

\section{ARROW microstuctured optical fiber based sensor}

In this section, we propose all-fiber based sensor that utilizes high sensitivity of the positions of the transmission spectrum minima to the refractive index of the material infiltrated into the air holes. The analytical model described in the previous section and numerical simulations using two- and three-dimensional beam propagation method [35] are used for design and characterization of the device.

Schematics of ARROW-MOF-based sensors are shown in Fig. 3. Light is guided by low-index silica core. The analyte is infiltrated into the air-holes. The sensor is used to distinguish between the analytes with slightly different refractive indexes. The difference in their refractive indexes can be due to different characteristics. For example, it can reflect the difference in the concentration (c) of a solute as illustrated in Fig. 3a. Another possibility corresponds to the case when the change in the refractive index of the analyte reflects the change in the temperature (t) of the surrounding medium (Fig. 3b). Potential advantages of this type of sensor include: (i) operation of the proposed device is based on a shift in the transmission spectrum induced by a change of the analyte's refractive index (as shown in Fig. 3c) implying that the absolute value of transmission does not need to be taken into account thus eliminating the requirement for a separate reference channel; (ii) the proposed sensor is a simple allfiber compact device realizable with standard MOF drawing techniques. (iii) very small analyte volumes are required; (iv) a wide range of refractive index changes can be measured. Since the ARROW regime applies only in the case when $n>n_{0}$, a limitation associated with this type of sensor implemented in silica MOF is the requirement for the refractive index of the analyte to be larger than that of silica $(n>1.44)$.

As an example, we consider temperature sensor performance utilizing ARROW-MOF infiltrated with high-index liquid whose refractive index changes with the temperature

(a)

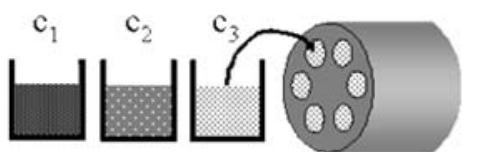

(b)

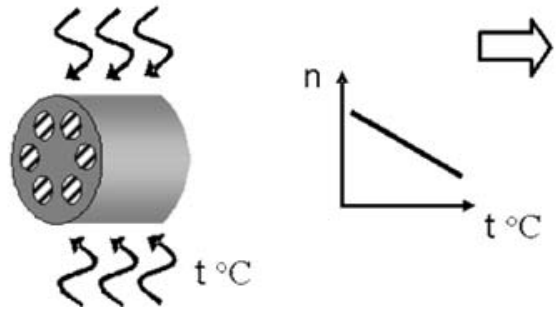

(c)

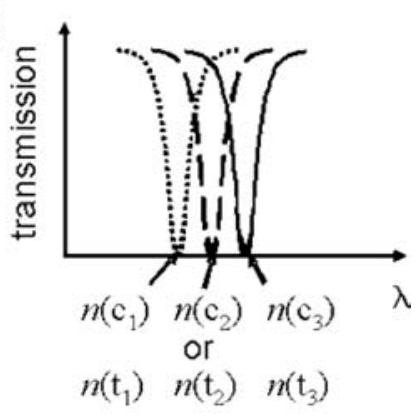

FIGURE 3 Schematics of ARROW-MOF-based sensors for concentration measurements $(n(\mathrm{c})) \mathbf{a}$; temperature measurements $(n(\mathrm{t}))$ b. Transmission minimum wavelength shift as a function of a refractive index of an analyte $\mathbf{c}$ 
as $\mathrm{d} n(t) / \mathrm{d} t=-\alpha /{ }^{\rho} \mathrm{C}$. For simplicity, we assume that both $n_{0}$ and $n$ do not change with wavelength. The following parameters have been used in our simulations: silica core refractive index $n_{0}=1.44$, propagation length $L=1 \mathrm{~cm}$, core size $D=12.2 \mu \mathrm{m}$, air-hole diameter $d_{\mathrm{pl}}=3.62 \mu \mathrm{m}$, lattice constant $\Lambda=8 \mu \mathrm{m}$, and the refractive index of the analyte $n\left(t_{0}\right)=1.8$, that is specified at a given temperature $t_{0}$. Refractive index profile for the planar equivalent of MOF is shown in Fig. 1b. Resonant wavelength shift $\delta \lambda_{\mathrm{r}}=\lambda_{\mathrm{r}}\left(n\left(t_{0}\right)\right)-\lambda_{\mathrm{r}}\left(n\left(t_{1}\right)\right)$ as a function of the refractive index change $\delta n=n\left(t_{0}\right)-n\left(t_{1}\right)$ is shown in Fig. 4 for several resonant orders of ARROWMOF $(m)$. Lines correspond to the analytical predictions (using Eq. (1)) and dots correspond to the numerical simulations. As shown in Fig. 4, sensitivity of the sensor $R=\partial \lambda_{\mathrm{r}} / \partial n$ increases as the resonant order $m$ decreases. This result is consistent with the predictions of Eqs. (1) and (2). Also, as it follows from the same equations, the sensor sensitivity is proportional to the width (diameter) of the high-index layer (cylinder). On the other hand, as the resonance order decreases, the width of low transmission region increases [28]. As a result the transmission minima corresponding to refractive indexes at temperatures $t_{1}$ and $t_{2}$ may overlap thus reducing the resolution of the sensor. Therefore, there is a trade-off between the sensitivity and the resolution that has to be taken into account to optimize the sensor design.

Figure 5a shows an example of a sensor operating at $m=10$ resonance with the parameters used in Fig. 4 for two values of refractive index change, $\delta n=0.0025$ and $\delta n$ $=0.005$. From our simulations, we find a wavelength shift of $3 \mathrm{~nm}$ corresponding to refractive index change of $\delta n=$ 0.0025 . This result is in good agreement with the analytical predictions from Eq. (1).

In order to make a transition from the two-dimensional waveguide to the three-dimensional MOF, a simple formula relating the width of the high-index layer of the planar structure, $d_{\mathrm{pl}}$, and the diameter of the high-index inclusion in MOF, $d_{\mathrm{MOF}}$, can be used $d_{\mathrm{MOF}}=d_{\mathrm{pl}}(m+1 / 2) / m$. Note that this formula relates $d_{\mathrm{pl}}$ and $d_{\mathrm{MOF}}$ at a particular resonance $m$. We use this formula for designing a MOF-based sensor.

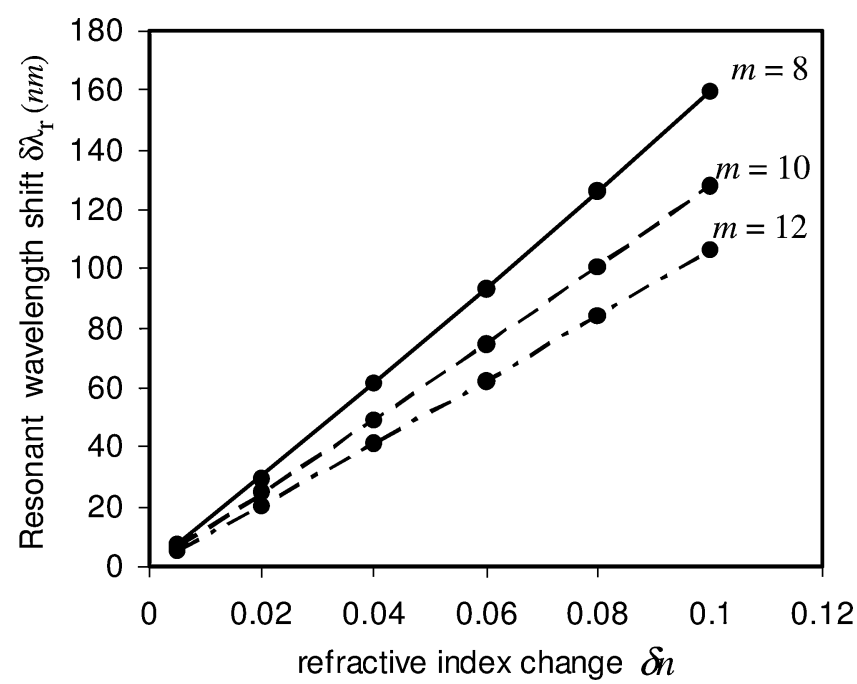

FIGURE 4 ARROW resonant wavelength shift as a function of the change in the refractive index of the analyte for different resonant orders $m$
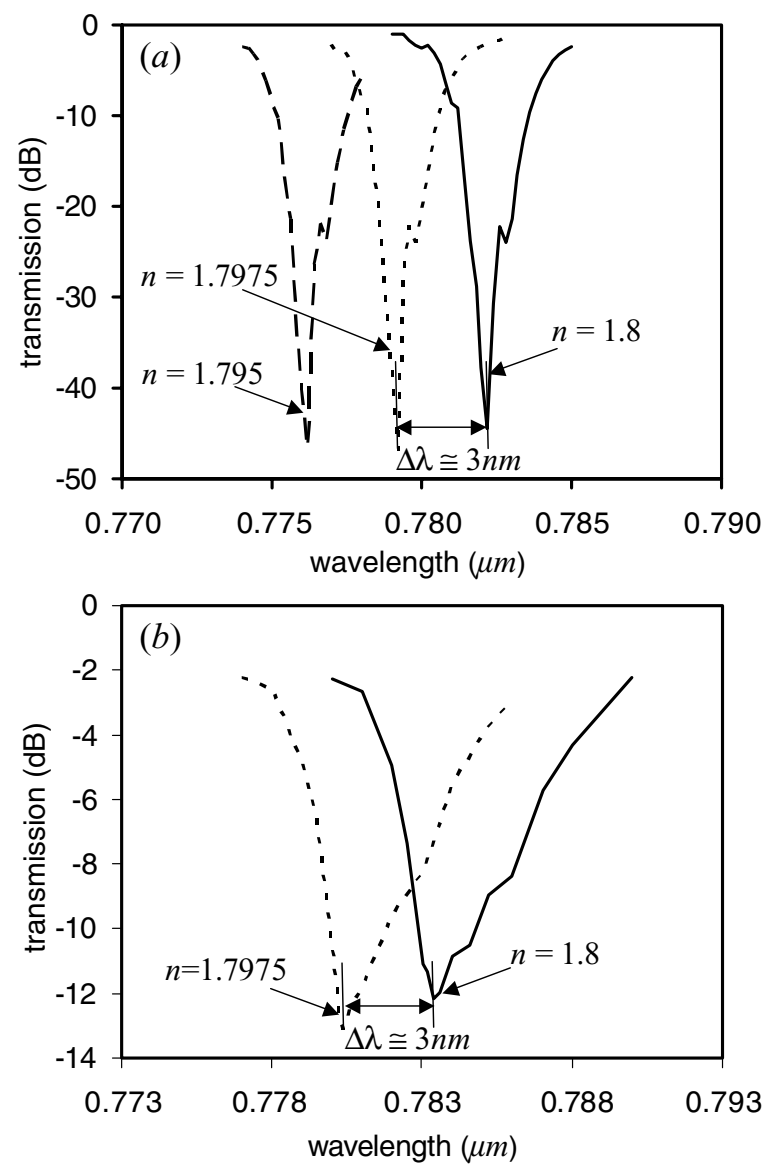

FIGURE 5 Calculated transmission spectra near the $m=10$ resonance for two-dimensional structure of length $L=1 \mathrm{~cm}$ with three values of the refractive index of the analyte $(1.8,1.7975,1.795 \mathbf{a}$, three-dimensional structure of length $L=1024 \mu \mathrm{m}$ with two values of the refractive index of the analyte $(1.8,1.7975) \mathbf{b}$

We performed three-dimensional simulations using beam propagation method [35] for two values of the refractive index of the analyte that differ by $\delta n=0.0025$. These simulations allow us to estimate the length of the actual MOF-based device and therefore, the volume of the analyte that is required. Figure $5 \mathrm{~b}$ shows the spectral shift of the transmission minimum corresponding to $m=10$ resonance of ARROWtype MOF. Consistent with the predictions of the Eq. (2), $\Delta \lambda \cong 3 \mathrm{~nm}$. The propagation length used in these simulations was $L=1024 \mu \mathrm{m}$, air-hole size $d_{\mathrm{MOF}}=3.8 \mu \mathrm{m}$, lattice constant $\Lambda=8 \mu \mathrm{m}$, refractive indexes of the analyte 1.7975 and 1.8 as shown in Fig. 5. The results show that propagation length of $\sim 1 \mathrm{~mm}$ in the MOF consisting of two rings of air holes filled with the analyte is sufficient to detect the refractive index difference of $\sim 0.1 \%$ and therefore, very small analyte volumes $\sim \mathrm{nL}$ are required for this type of sensor. Thus, very compact devices utilizing small analyte volumes can be realized using ARROW-type of MOFs.

Finally, the ARROW-MOF-based sensor can be potentially useful for humidity and gas sensing if the air-holes are infiltrated with a polymer, which reversibly changes its refractive index upon exposure to a water vapor or to a specific gas, respectively. Examples of such polymers are polyethylene oxide used for humidity sensing [36] and ferrocene-based 
polymers used for various gases sensing [37]. The refractive indexes of these polymers are higher than that of the silica fiber and therefore can be used in ARROW structure. However, some practical challenges associated with uniformity of the refractive index distribution inside the inclusions may be anticipated and should be further explored in a particular experimental design.

4

\section{Summary}

We reviewed unique transmission properties of a class of microstructured fibers, ARROW-type MOFs, and applied them for designing a novel refractometric sensor. Analytical predictions and numerical analysis were utilized to characterize the sensor performance including the sensitivity, measurement range and design trade-offs. In our design the analyte is placed inside the air holes of the microstructured optical fiber and operation of the sensor is based on the shift in the transmission spectrum induced by the change of the analyte's refractive index. Sensitivity of the proposed sensor was studied as a function of resonant wavelength order. The advantages of using ARROW-type MOF include good sensitivity over a wide range of refractive index changes, very small analyte volumes required for detection and simple design rules that follow from the analytical model for the resonant wavelengths of ARROW structure. The technique has been shown to allow detection the refractive index change of $\delta n \sim 10^{-3}$.

ACKNOWLEDGEMENTS The authors thank Paul Steinvurzel for helpful discussions on numerical simulations.

\section{REFERENCES}

1 E. Udd, Fiber Optic Sensors: An Introduction for Engineers and Scientists (John Wiley \& Sons. NY 1991)

2 A.G. Mignani, F. Baldini, Rep. Prog. Phys. 59, 1 (1996)

3 S.W. James, R.P. Tatam, Meas. Sci. Technol. 14, R49 (2003)

4 J.C. Knight, J. Broeng, T.A. Birks, P. St. J. Russell, Science 282, 1476 (1998)

5 R.F. Cregan, B.J. Mangan, J.C. Knight, T.A. Birks, P. St. J. Russell, P.J. Roberts, D.C. Allan, Science 285, 1537 (1999)

6 J.C. Knight, Nature 424, 847 (2003)

7 P.St.J. Russell, Science 299, 358 (2003)

8 J. Fini, R. Bise, Jpn. J. Appl. Phys. 43, 5717 (2004)
9 T.M. Monro, D.J. Richardson, P.J. Bennett, Electron. Lett. 35, 1188 (1999)

10 T.M. Monro, W. Belardi, K. Furusawa, J.C. Baggett, N.G.R. Broderick, D.J. Richardson, Meas. Sci. Technol. 12, 854 (2001)

11 Y.L. Hoo, W. Jin, H.L. Ho, D.N. Wang, R.S. Windeler, Opt. Eng. 41, 8 (2002)

12 Y.L. Hoo, W. Jin, C. Shi, H.L. Ho, D.N. Wang, S.C. Ruan, Appl. Opt. 42, 3509 (2003)

13 R. T. Bise, D. J. Trevor, Proc. OFC, WI4 (2004)

14 J.M. Fini, Meas. Sci. Technol. 15, 1120 (2004)

15 T. Ritari, J. Tuominen, H. Ludvigsen, J.C. Petersen, T. Sørensen, T.P. Hansen, H.R. Simonsen, Opt. Express 12, 4080 (2004)

16 R.T. Bise, R.S. Windeler, K.S. Kranz, C. Kerbage, B.J. Eggleton, D.J. Trevor, in OSA Trends in Optics and Photonics (TOPS) vol. 70, Optical Fiber Communication Conference, Technical Digest, post conference edition (Optical Society of America, Washington DC, 2002), p 466

17 N.M. Litchinitser, S.C. Dunn, B. Usner, B.J. Eggleton, T.P. White, R.C. McPhedran, C. Martijn de Sterke, Opt. Express 11, 1243 (2003)

18 N.M. Litchinitser, S.C. Dunn, P.E. Steinvurzel, B.J. Eggleton, T. P. White, R.C. McPhedran, C.M. de Sterke, Opt. Express 12, 1540 (2004)

19 P. Steinvurzel, B.T. Kuhlmey, T.P. White, M.J. Steel, C.M. de Sterke, B.J. Eggleton, Opt. Express 12, 5424 (2004)

20 T.T. Larsen, A. Bjarklev, D.S. Hermann, J. Broeng, Opt. Express 11, 2589 (2003)

21 T.T. Alkeskjold, J. Lægsgaard, A. Bjarklev, D.S. Hermann, A. Anawati, J. Broeng, J. Li, S. Wu, Opt. Express 12, 5857 (2004)

22 J. Laegsgaard, J. Opt. A, Pure and Appl. Opt. 6, 798 (2004)

23 F. Luan, A.K. George, T.D. Hedley, G.J. Pearce, D.M. Bird, J.C. Knight, P.St.J. Russell, Opt. Lett. 20, 2369 (2004)

24 A. Argyros, T.A. Birks, S.G. Leon-Saval, C.B. Cordeiro, F. Luan, Russell, Opt. Express 13, 309 (2005)

25 A. Argyros, T.A. Birks, S.G. Leon-Saval, Cordeiro, P.St.J. Russell, Opt. Express 13, 2503 (2005)

26 J. Jasapara, T.H. Her, R. Bise, R. Windeler, D.J. DiGiovanni, J. Opt. Soc. Am. B20, 1611 (2003)

27 N.M. Litchinitser, A.K. Abeeluck, C. Headley, B.J. Eggleton, Opt. Lett. 27, 1592 (2002)

28 A.K. Abeeluck, N.M. Litchinitser, C. Headley, B.J. Eggleton, Opt. Express 10, 1320 (2002)

29 T.P. White, R.C. McPhedran, C. Martijn de Sterke, N.M. Litchinitser, B.J. Eggleton, Opt. Lett. 27, 1977 (2002)

30 Cargille Laboratories, Inc., NJ, USA, www.cargille.com

31 M.A. Duguay, Y. Kokubun, T.L. Koch, L. Pfeiffer, Appl. Phys. Lett. 49, $13(1986)$

32 E.A.J. Marcatili, R.A. Schmeltzer, Bell Syst. Tech. J. 43, 1783 (1964)

33 T. Baba, Y. Kokubun, IEEE J. Quantum Electron. 28, 1689 (1992)

34 J.-L. Archambault, R.J. Black, S. Lacroix, J. Bures, J. Lightwave Technol. 11, 416 (1993)

35 R. Scarmozzino, A. Gopinath, R. Pregla, S. Helfert, IEEE J. Select. Topics Quantum Electron. 6, 150 (2000)

36 D.C. Bownass, J.S. Barton, J.D.C. Jones, Opt. Lett. 22, 346 (1997)

37 M. Shadaram, J. Martinez, F. Garcia, D. Tavares, S. Arnand, N. Godbout, S. Lacroix, Proc. SPIE 2695, 164 (1996) 